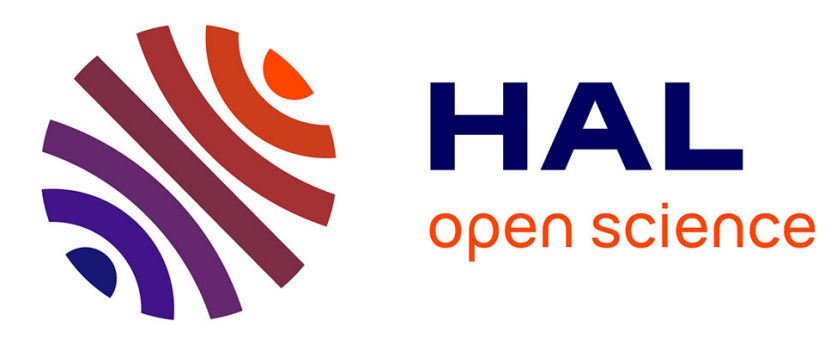

\title{
Van Hove singularities and trap states in two-dimensional CdSe nanoplatelets
}

Nemanja Peric, Yannick Lambert, Shalini Singh, Ali Hossain Khan, Nathali Alexandra Franchina Vergel, D. Deresmes, Maxime Berthe, Zeger Hens, Iwan Moreels, Christophe Delerue, et al.

\section{To cite this version:}

Nemanja Peric, Yannick Lambert, Shalini Singh, Ali Hossain Khan, Nathali Alexandra Franchina Vergel, et al.. Van Hove singularities and trap states in two-dimensional CdSe nanoplatelets. Nano Letters, 2021, 21 (4), pp.1702-1708. 10.1021/acs.nanolett.0c04509 . hal-03152599

\section{HAL Id: hal-03152599 https://hal.science/hal-03152599}

Submitted on 23 Aug 2021

HAL is a multi-disciplinary open access archive for the deposit and dissemination of scientific research documents, whether they are published or not. The documents may come from teaching and research institutions in France or abroad, or from public or private research centers.
L'archive ouverte pluridisciplinaire HAL, est destinée au dépôt et à la diffusion de documents scientifiques de niveau recherche, publiés ou non, émanant des établissements d'enseignement et de recherche français ou étrangers, des laboratoires publics ou privés. 


\title{
Van Hove singularities and trap states in two- dimensional CdSe nanoplatelets
}

\author{
Nemanja Peric ${ }^{\dagger}$, Yannick Lambert ${ }^{\dagger}$, Shalini Singh ${ }^{\ddagger}$, Ali Hossain Khan ${ }^{\ddagger}$, Nathali Alexandra \\ Franchina Vergel ${ }^{\dagger}$, Dominique Deresmes ${ }^{\dagger}$, Maxime Berthe ${ }^{\dagger}$, Zeger Hens ${ }^{\dagger}$, Iwan Moreels ${ }^{\dagger}$, \\ Christophe Delerue ${ }^{\dagger}$, Bruno Grandidier $^{\dagger} *$, and Louis Biadala ${ }^{\dagger} *$ \\ ${ }^{\dagger}$ Univ. Lille, CNRS, Centrale Lille, Univ. Polytechnique Hauts-de-France, Junia-ISEN, Centrale \\ Lille, UMR 8520 - IEMN, F-59000 Lille, France \\ Physics and Chemistry of Nanostructures, Ghent University, 9000 Ghent, Belgium
}

\begin{abstract}
Semiconductor nanoplatelets, which offer a compelling combination of the flatness of twodimensional semiconductors and the inherent richness brought about by colloidal nanostructure synthesis, form an ideal and general testbed to investigate fundamental physical effects related to the dimensionality of semiconductor. With low temperature scanning tunnelling spectroscopy and tight binding calculations, we investigate the conduction band density of states of individual CdSe nanoplatelets. We find an occurrence of peaks instead of the typical step-like function associated with a quantum well, that rule out a free in-plane electron motion, in agreement with the theoretical density of states. This finding, along with the detection of deep trap states located
\end{abstract}


on the edge facets, which also restrict the electron motion, provides a detailed picture of the actual lateral confinement in quantum wells with finite length and width.

KEYWORDS: CdSe nanoplatelets, nanocrystals, quantum confinement, dimensionality, scanning tunneling spectroscopy, traps. 
In condensed matter physics, a stepwise reduction of the dimensionality from bulk to the nanoscale is known to lead to striking electronic and optical properties such as the giant oscillator strength transition for two-dimensional (2D) semiconductor quantum wells ${ }^{1}$ and the spin-charge separation in one-dimensional (1D) systems. ${ }^{2}$ However, in contrast to metals, the lateral confinement in semiconductor materials competes with the exciton Coulomb interaction, which can be magnified by dielectric mismatch. Due to the complexity of these interactions, ${ }^{3}$ the degree of freedom in the electron motion remains poorly understood, whereas its clear circumscription could significantly improve the optoelectronic performances of quasi-2D systems such as CdSe colloidal nanoplatelets (NPLs).

Since the distribution of the quantized states, the so-called density of states (DOS), reflects the restrictions of the electron motion with dimensionality, it can serve as a genuine fingerprint to unambiguously disclose the confinement experienced by the charge carriers. A unique way to probe the DOS consists in measuring single-particle excitation spectra with scanning tunnelling spectroscopy $(\mathrm{STS})^{4-7}$. Here we used this technique to study electron confinement in colloidal CdSe nanoplatelets (NPLs), which have a quantized thickness $d$ due to a discrete number of monolayers (ML) and a rectangular shape with finite length $L$ and width $W$. Recently, Ji et al. have demonstrated that the hole DOS is consistent with a free in-plane motion, while the electrons have a more complex DOS that deviates from the simple 2D model ${ }^{8}$. When $W$ or/and $L$ are smaller than ten to five times the exciton Bohr radius, the electron behavior, which has a smaller effective mass than the hole, becomes affected, with a significant impact on its interaction with the hole and the dielectric image charges. The already present complexity in understanding electron-hole correlation in these box-shaped nanostructures is further enhanced with the existence of electrical surface traps, which are caused by the sporadic absence of 
ligands. It makes the electron confinement in the NPLs still unknown, leading to intense debates about their true dimensionality. ${ }^{9,10}$

In this study, two sets of CdSe NPLs capped with oleate ligands having thicknesses of 5.5 $\operatorname{ML}\left(d_{5.5}=1.65 \mathrm{~nm}\right)$ and $7.5 \mathrm{ML}\left(d_{7.5}=2.25 \mathrm{~nm}\right)$ were synthesized. ${ }^{11,12}$ While transmission electron microscopy (TEM) shows the overall rectangular shapes of the NPLs and yields their lateral sizes, $(21 \pm 2) \times(7 \pm 1) \mathrm{nm}^{2}$ for the 5.5 ML-thick NPLs, $(45 \pm 10) \times(9 \pm 1) \mathrm{nm}^{2}$ for the 7.5 ML-thick NPLs (Figure 1a and Figure S1), the thickness of the NPLs is directly obtained from their optical properties. The optical absorption spectra measured at room temperature are dominated by two absorption lines (Figure 1d), attributed to the formation of excitons composed of the $1 \mathrm{~S}$ electron $\left(\mathrm{e}_{1}\right)$ in the conduction band $(\mathrm{CB})$ and the $1 \mathrm{~S}_{3 / 2}$ heavy holes (hh) and light holes (lh) in the valence band. ${ }^{13}$ At room temperature, the photoluminescence (PL) spectra consist of a single sharp Lorentzian line from the radiative recombination of hh excitons (Figure 1e). As the NPL thickness decreases, the hh exciton energy steadily increases because of stronger vertical quantum confinement. ${ }^{13}$ The additional feature observed at $2.13 \mathrm{eV}$ in the PL spectra of 7.5 ML NPLs is due to the presence of a small fraction of 6.5 ML NPLs. At cryogenic temperature (Figure 1f), the PL spectra of the NPLs exhibit an emission doublet, with decreasing separation for larger NPL thicknesses, which is attributed to the radiative recombination of the hh exciton (high energy peak) and its negative trion (low energy peak). ${ }^{14}$ The PL spectra measured at $\mathrm{T}=$ 4.2 $\mathrm{K}$ reveals the presence of a small and broad band in an energy range extending below the band gap energy. It is attributed to the recombination through trap states in the band gap,${ }^{15}$ which are caused by the incomplete passivation of surface dangling bonds (DB) with organic ligands.

While insightful, optical transitions do not provide a direct access to the energy levels of the NPLs. In particular, the relative positions of the trap states with respect to the Fermi level 
remains unknown. To this aim, the NPLs were drop-casting on gold surfaces to be studied with STS. Their arrangement was first observed with scanning electron microscopy (SEM) and atomic force microscopy (AFM). As shown in Figures $1 \mathrm{~b}$ and $\mathrm{c}$, the 5.5 ML NPLs stack to form columnar assemblies. Only a small number of 5.5 ML NPLs adsorb in a flat-lying geometry. For this configuration, the apparent height measured from the scanning tunnelling microscopy (STM) image yields $4.2 \mathrm{~nm}$ (Figure $2 \mathrm{~b}$ and c). This value is consistent with a NPL thickness of $1.65 \mathrm{~nm}$ surrounded by tilted oleate ligands (around $2 \mathrm{~nm}$ in length) on top and bottom Cd-rich (100) facets. In contrast to the 5.5 ML NPLs, the majority of the 7.5 ML NPLs were found flat on the Au surface (Figure S2). We attribute this change to dichloromethane that was used as a solvent for the 7.5 ML NPLs to reduce clustering effects.

For the sake of clarity in the data interpretation, tunnelling spectroscopic measurements were first acquired on the isolated and flat-lying NPLs (Figure 2a), which show the typical configuration of resonant tunneling across a double tunneling junction. In this geometry, by choosing tunnelling currents smaller than 100 pA as setpoints, the width of the STM tip-NPL junction is usually larger than the one of the NPL-gold junction and limits the current. In such a situation, where an electron tunneling from the tip to the NPL leaves the NPL before a second electron arrives, the zero-conductance region (ZCR), surrounded by peaks at positive and negative sample voltages, gives access to the quasiparticle band gap of the NPLs. ${ }^{16,17}$ The peaks occur at voltages that correspond to the energy levels of the NPLs plus the polarization energy of the charge carriers divided by the capacitance ratio of the two junctions, the so-called lever arm. ${ }^{18}$ Examination of the ZCRs in Figures $2 \mathrm{~d}$ and e shows larger apparent band gaps for thinner NPLs, with values consistent with those measured in Ref. 8. While the lever arm is close to one for the 7.5 ML NPLs with partially chloride-passivated facets, smaller lever arms of $0.80 \sim 0.85$ 
are occasionally found for 5.5 ML NPLs, due to the smaller in-plane dimensions and the thicker NPL-gold junction consisting of long oleic acid ligands only. For these few spectra exhibiting apparent band gaps much larger than the optical gap, two neighboring peaks, separated by 200 $\mathrm{mV}$, are clearly seen at negative bias (Figure 2d, 5.5 ML NPL). They are attributed to the heavy hole (hh) and light hole/split-off (lh/so) states, the mixing between the lh and so bands arising from a 2D confinement. ${ }^{8}$ Most importantly, whatever the size of the NPLs that are probed, the STS measurements consistently reveal the presence of one or several peaks in CB, in agreement with a recent STS work. ${ }^{8}$ This behaviour clearly depart from the typical step-like function observed in the tunneling spectra of semiconductor quantum wells. ${ }^{7,19}$ Its observation rules out the simple picture of an electron moving freely in two dimensions.

We now turn to the tunnelling spectra measured on the stacks. As shown in the STM topography of Figure 3a, the NPLs are individually resolved within a stack. An average separation of $4.3 \pm 0.1 \mathrm{~nm}$ between the NPLs (Figure $3 \mathrm{~b}$ ) suggests an entwinement of the ligands for the largest facets which are face-to-face. The thickness of the whole junction reaches about $7.5 \mathrm{~nm}$ in STM, in agreement with the average width of the NPLs and a disordered layer of ligands on the side facets (inset Figure 3c). Based on the height profiles (see also Figure S2), the NPLs lie along their longest edge,$^{20,21}$ providing another access to the DOS from the side facets. Tunneling spectra fall into two classes. Some NPLs show a ZCR similar to the one observed for the flat-lying NPLs (Figure 3c). In this case, the gap is surrounded by an intense peak at positive bias and a doublet peak at negative bias. As the ZCR increases with higher setpoint current, ${ }^{18,22,23}$ we unambiguously identify the intense peak as an electron level in $\mathrm{CB}$ and the doublet peak in $\mathrm{VB}$ as the hh and $\mathrm{lh} / \mathrm{so}$ states. Notice that the peaks in $\mathrm{CB}$ are usually more intense in comparison with the peaks found for the flat-lying NPLs, while the apparent band gap in 
unchanged, as discussed in the Supporting Information. In other cases, the spectra still exhibit the highly intense peak at positive bias, yet the ZCR is inconsistently smaller than the optical gap of the 5.5 ML NPLs. For example, in Figure 3d, the ZCR is a mere $1.63 \mathrm{eV}$, while the optical gap measured in Figure 1f was $2.33 \mathrm{eV}$. Such a behavior was not seen for the flat-lying NPLs. Moreover, recording hundreds of consecutive spectra over 30 minutes with the same feedback parameters reveals the occurrence of sudden shifts in the onset of the current at positive bias (Figure S3). This result mirrors the typical spectral diffusion phenomenon obtained on the PL emission linewidth of individual nanocrystals attributed to charge fluctuation in the local environment of the $\mathrm{NCs}^{24,25}$ Altogether, these results point toward the presence of states within the band gap of CdSe NPLs (Figure S4).

In the literature, the trap states at the surface of colloidal semiconductor nanocrystals have been designated as the main source of in-gap states. ${ }^{26,27}$ These trap states stem from the DBs of unpassivated atoms at the nanoparticle surface. For CdSe nanocrystals, it has been predicted that selenium DBs at the surface of the nanocrystal would be the main source of electrical traps. ${ }^{11,28,29}$ In contrast with spherical nanocrystals, NPL facets are very well defined upon their orientation. Top and bottom facets are pure $\mathrm{Cd}\{100\}$ facets, while the side-facets can consist of Se rich facets. Here, the comparison between the spectra acquired on the flat-lying NPLs and those acquired on NPL within stacks indicates that traps states are only detected when NPLs rest on their edge. This finding is consistent with conclusions drawn from macroscopic analyses of the NPLs, which pinpointed a preferred localization of trap states at the NPL edges. On these facets, the ligands are the most labile and their desorption favours the formation of 2coordinated selenium surface atoms. ${ }^{11,28}$ The nature of the DBs in CdSe NPLs, is further supported by the relative energetic position of the trap state in the band gap. These deep states, 
that lay below the Fermi level, are found almost midgap, in agreement with DFT calculations assuming Se DBs. ${ }^{11}$

When tunnelling charge carriers are captured or released by a deep level, clear stepwise changes occur in single real-traces signatures of charging or discharging events (Figure S3). These sudden variations of the tunnelling current are seen at positive bias only and they are found to be distributed over a few hundreds of meV. Several charging events can even take place during the same trace. These charges dramatically affect the potential distribution between the tip and the Au surface, which has been shown to result in a substantial shift of the CB states. ${ }^{30,31}$ Similar to Ref.30 we attribute a sudden decrease of the current to the release of one electron by a trap state to the gold substrate, since the next trace shows a clear shift towards smaller positive bias. Starting with $N$ excess of electrons, the NPL loses one electron and changes its charge state to $N$-1. As a result, a larger portion of the $\mathrm{CB}$ becomes accessible. For electrostatic shifts exceeding $500 \mathrm{meV}$, the differential conductance unveils a wealth of features that, again, are not compatible with the ones of a 2D quantum well.

In order to get deeper insight into these features, tight binding calculations were performed to determine the CB structure of the NPLs (see Supporting Information). Starting with the geometry of an infinitely long NPL and a finite width $(W=6 \mathrm{~nm})$, the calculations yield the typical DOS of a quantum wire for any NPL thickness from 2.5 ML to 8.5 ML (Figure 4a). It consists of Van Hove singularities, that can be gathered within subbands. The transitions between the lowest subbands are clearly identified when the band structure is plotted in the Brillouin zone (Figure S5). They correspond to a change of the peak intensity and spacing in the DOS. As expected, the position of the first peak, which corresponds to the lowest subband, shifts 
towards higher energy as the thickness of the NPL decreases because of strong confinement (Figure 4a).

In contrast, the energy difference between subsequent peaks is directly linked to the width of the NPL (Figure 4b). For increasing widths and consequent reduction of lateral confinement, the peak spacing decreases, revealing a step-like function modulated by closer and closer singularities. Ultimately, the DOS converges to a pure step-like function, the fingerprint of a quantum well. Based on our calculations, a peak spacing of the order of $25 \mathrm{meV}$ is obtained for the lowest subbands of infinitely long NPLs with a width of $30 \mathrm{~nm}$. While such NPLs could be approximated as 2D systems at room temperature, the NPLs that are considered here, and more generally in the literature, definitely deviate from this simple picture. The tight binding calculations also reveal a strong influence of the NPL length. Reducing the length to realistic lengths of 20-30 nm leads to the formation of oscillations in the DOS between the reciprocal square root singularities (Figure 4c). This feature indicates the loss of a free electron motion in the plane of the NPLs. As the motion becomes more and more restricted, the 1D DOS vanishes in favour of an oscillating DOS. Therefore, the van Hove singularities and the strength of the oscillating DOS are the signature of the electron behaviour in the NPLs: their respective contribution to the DOS provides a direct insight into the lateral extend of the electron wave function.

In order to compare the theoretical DOS with experiments, we selected NPLs, where tens of successive traces were measured without any stepwise changes of the current at positive bias. This condition ensures the absence of any significant electrostatic change in the environment of the NPLs. Two examples of spectra acquired on the stacked 5.5 ML NPLs are shown in Figure 4d. They exhibit both features of the calculated DOS: the singularities superimposed to a small 
oscillation. Note that the energy difference between the maxima of amplitude in the small oscillation is too large to be attributed to a polaron contribution of optical phonons (26 meV for $\mathrm{CdSe}$ ) as measured on nanocrystals. ${ }^{32}$ Instead, the experimental DOS observed here indicates a large delocalization of the electron wave function in the NPLs, where the boundaries of the NPLs act as potential barriers that laterally confine the electron wave function.

Notwithstanding the qualitative agreement with the experimental results, the tight binding calculations quantitatively reproduce the experimental data for realistic dimensions of NPLs $(d=$ 5.5 ML; $W=5 \mathrm{~nm} ; L=30 \mathrm{~nm}$ ). As the calculations do not include any corrections for the selfenergy, our results suggest a weak self-energy of the tunnelling electron. We explain this effect by the substantial dielectric screening that arises from the proximity of the NPLs in the stack and the presence of top and bottom metal electrodes. This quantitative agreement further emphasizes the structural quality of NPLs: any localization potential, scattering with phonons, or defect in the lattice would dramatically alter the DOS by notably inducing a strong broadening or even the vanishing of the Van Hove singularities. We note however that the degree of delocalization must be slightly alleviated. The agreement between the experimental spectra and the theoretical DOS shown in Figure $4 \mathrm{~d}$ is only obtained when comparing the DOS of $5 \mathrm{~nm}$ wide NPLs with STS spectra recorded on $7 \mathrm{~nm}$ wide NPLs. This difference indicates that the electron wave function does not fully extend up to the boundaries. Despite the presence of traps on the edge of the NPLs that modify the confining potential when they are negatively charged, we assign the existence of the small depleted layer along the side-facets of the NPLs to a dipole layer arising from ligands and/or surface reconstruction, which can account for a very localized potential. ${ }^{33}$

In summary, the CB structure of CdSe NPLs has been investigated with STS. The observation of Van Hove singularities imply a paradigm shift on the electronic structure of 
typical CdSe NPLs considered in the literature. As the electron DOS exhibits a striking modulation that is directly related to the length of the NPLs, delineating the in-plane electron motion at low temperature has important consequences for a deeper understanding of the exciton dissociation, diffusive transport and annihilation in NPLs. Moreover, it is shown that the sidefacets of NPLs host electronic deep trap states, which cause Coulomb blockade in the tunnelling current. As they could be fully removed by the formation of core-crown NPL, our results anticipate a genuine boost for NPL-based lateral heterostructures that will notably allow for the mixing of the dimensionalities to favour specific electronic or optical properties. 
FIGURE 1. a) TEM, b) SEM and c) AFM images of 5.5 ML-thick NPLs deposited on Au surface. d) Absorption and e) photoluminescence spectra at room temperature of 5.5 and 7.5 ML NPLs in solution. f) Photoluminescence spectra at $\mathrm{T}=4.2 \mathrm{~K}$ of 5.5 ML and 7.5 ML NPLs drop casted on glass substrate. Spectra are offset for clarity. The dashed line indicates the baseline for 7.5 ML. The contribution of 6.5 ML NPLs in the PL spectrum is enhanced by a factor of 6 for clarity. The arrow indicates the deep trap emission in the PL spectra of 5.5 ML NPLs.

FIGURE 2. a) Illustration of the STS on individual flat-lying NPL. b) STM image of two flatlying CdSe NPLs with a thickness of 5.5 ML and c) corresponding height profiles. Tunneling conditions: $\mathrm{V}_{\text {sample }}=-1.5 \mathrm{~V}, \mathrm{I}_{\mathrm{t}}=5 \mathrm{pA}$. STS of a 7.5 ML NPL (black) and a 5.5 ML NPL (red) measured at d) $\mathrm{T}=77 \mathrm{~K}$ and e) $5 \mathrm{~K}$ respectively. The separation between the lowest peak in the conduction band and the highest peak in the valence band, labeled $\mathrm{e}_{1}$ and hh respectively, is indicated. The light hole/split-off state is labelled lh/so.

FIGURE 3. a) STM image of a stack of CdSe 5.5 ML NPLs. Tunneling conditions: $\mathrm{V}_{\text {sample }}=-5.0$ $\mathrm{V}, \mathrm{I}_{\mathrm{t}}=10 \mathrm{pA}$. b) (top) Height profile measured at the position of the horizontal segment in a), across a larger area image than the one shown in the STM image. (Bottom) Height profile on top of the stack pointing out the inter NPL distance. c) STS of a trap-free NPL within a stack measured at different setpoint currents as indicated in the graph. Feedback voltage: $\mathrm{V}_{\text {sample }}=-1.5$ V. Arrows point to the shift observed with increasing setpoint currents for the lowest peak in the conduction band $\mathrm{e}_{1}$ and the highest peaks in the valence band labeled hh and $\mathrm{h} / \mathrm{so}$, respectively. Inset: schematic of a stack of NPLs probed with a STM tip. d) Sequence of three differential conductance spectra acquired on a stacked NPL bearing a trap state and averaged over a few successive curves between charging and discharging events. $e_{1}$ indicates the position of the first 
electron subband, while the downward arrow indicates the highest trap state in the band gap of the NPL. The curves are offset for clarity. Feedback conditions: $\mathrm{V}_{\text {sample }}=-1.8 \mathrm{~V}, \mathrm{I}_{\mathrm{t}}=80 \mathrm{pA}$.

FIGURE 4. Theoretical conduction band density of states of CdSe nanoplatelets. a) DOS of infinitely long NPLs with a width of $6 \mathrm{~nm}$ and varying thicknesses. b) DOS of infinitely long NPLs with a fixed thickness of 5.5 ML and different widths. Due to the higher number of states contributing to the DOS for larger widths, the calculation becomes quickly limited to the lowest states. c) DOS of NPLs with a thickness of 5.5 ML, a width of $4 \mathrm{~nm}$ and different lengths. d) Comparison between two differential conductance spectra (black, labeled Exp.) and the theoretical DOS (red, labeled Theo.) of 5.5 ML NPLs with $\mathrm{L}=30 \mathrm{~nm}$ and $\mathrm{W}=5 \mathrm{~nm}$. Experimental conditions: $\mathrm{V}_{\text {sample }}=-1.8 \mathrm{~V}, \mathrm{I}_{\mathrm{t}}=100 \mathrm{pA}$, temperature $5 \mathrm{~K}$. The curves are offset for clarity.

\section{ASSOCIATED CONTENT}

\section{Supporting Information}

This material is available free of charge via the internet at http://pubs.acs.org. Methods for the synthesis, structural and optical analysis, STM experiments and tight binding calculations. Additional data (PDF).

\section{AUTHOR INFORMATION}

\section{Corresponding Author}

*E-mail: $\underline{\text { louis.biadala@iemn.fr, bruno.grandidier@iemn.fr }}$ 


\section{Author Contributions}

L.B. conceived and designed the experiments. A.K. and S.S. synthesized the NPLs, performed the TEM experiments and the room temperature optical characterization under the supervision of Z.H. and I.M. N.P. and L.B performed the STM measurements under the guidance of B.G and M.B. L.B and N.P. performed the optical characterization at cryogenic temperature. D.D. and Y.L. performed the AFM. N.F.V. and N.P. did the SEM experiments. C.D. performed the tight binding calculations. L.B., C.D. and B.G. interpreted the data. B.G. and L.B wrote the manuscript.

\section{ACKNOWLEDGMENT}

This study was financially supported by the European Community's H2020 Program (Grant No. PITN-GA-2016-722176, “Indeed” Project), the EQUIPEX program Excelsior (Grant No. ANR11-EQPX-0015), the RENATECH network, the Agence National de la Recherche (Grant No. ANR-19-CE09-0022, "TROPICAL" Project) and I-SITE ("PRIVET" project). This project has also received funding from the European Research Council (ERC) under the European Union's Horizon 2020 research and innovation programme (grant agreement No. 714876 PHOCONA). We also acknowledge the TEM facility of the Nematology Research Unit, member of the UGent TEM-Expertise center (life sciences).

\section{REFERENCES}

(1) Naeem, A.; Masia, F.; Christodoulou, S.; Moreels, I.; Borri, P.; Langbein, W. Giant Exciton Oscillator Strength and Radiatively Limited Dephasing in Two-Dimensional Platelets. Phys. Rev. B 2015, 91 (12), 121302. 
(2) Jompol, Y.; Ford, C. J. B.; Griffiths, J. P.; Farrer, I.; Jones, G. A. C.; Anderson, D.; Ritchie, D. A.; Silk, T. W.; Schofield, A. J. Probing Spin-Charge Separation in a Tomonaga-Luttinger Liquid. Science. 2009, 325 (5940), 597-601.

(3) Failla, M.; García Flórez, F.; Salzmann, B. B. V.; Vanmaekelbergh, D.; Stoof, H. T. C.; Siebbeles, L. D. A. Observation of the Quantized Motion of Excitons in CdSe Nanoplatelets. Phys. Rev. B 2020, 102 (19), 195405.

(4) Banin, U.; Cao, Y. W.; Katz, D.; Millo, O. Identification of Atomic-like Electronic States in Indium Arsenide Nanocrystal Quantum Dots. Nature 1999, 400 (6744), 542-544.

(5) Urbieta, A.; Grandidier, B.; Nys, J. P.; Deresmes, D.; Stiévenard, D.; Lemaître, A.; Patriarche, G.; Niquet, Y. M. Scanning Tunneling Spectroscopy of Cleaved InAs/GaAs Quantum Dots at Low Temperatures. Phys. Rev. B - Condens. Matter Mater. Phys. 2008, $77(15), 155313$.

(6) Ma, D. D. D.; Lee, C. S.; Au, F. C. K.; Tong, S. Y.; Lee, S. T. Small-Diameter Silicon Nanowire Surfaces. Science 2003, 299 (5614), 1874-1877.

(7) Franchina Vergel, N. A.; Tadjine, A.; Notot, V.; Mohr, M.; Kouassi N'Guissan, A.; Coinon, C.; Berthe, M.; Biadala, L.; Sossoe, K. K.; Dzagli, M. M.; et al. Influence of Doping Level and Surface States in Tunneling Spectroscopy of an I n 0.53 G a 0.47 As Quantum Well Grown on p -Type Doped InP(001). Phys. Rev. Mater. 2019, 3 (9), 094604.

(8) Ji, B.; Rabani, E.; Efros, A. L.; Vaxenburg, R.; Ashkenazi, O.; Azulay, D.; Banin, U.; Millo, O. Dielectric Confinement and Excitonic Effects in Two-Dimensional 
Nanoplatelets. ACS Nano 2020, 14 (7), 8257-8265.

(9) Scott, R.; Heckmann, J.; Prudnikau, A. V.; Antanovich, A.; Mikhailov, A.; Owschimikow, N.; Artemyev, M.; Climente, J. I.; Woggon, U.; Grosse, N. B.; et al. Directed Emission of CdSe Nanoplatelets Originating from Strongly Anisotropic 2D Electronic Structure. Nat. Nanotechnol. 2017, 12 (12), 1155-1160..

(10) Brumberg, A.; Harvey, S. M.; Philbin, J. P.; Diroll, B. T.; Lee, B.; Crooker, S. A.; Wasielewski, M. R.; Rabani, E.; Schaller, R. D. Determination of the In-Plane Exciton Radius in 2D CdSe Nanoplatelets via Magneto-Optical Spectroscopy. ACS Nano 2019, 13 (8), 8589-8596.

(11) Singh, S.; Tomar, R.; ten Brinck, S.; De Roo, J.; Geiregat, P.; Martins, J. C.; Infante, I.; Hens, Z. Colloidal CdSe Nanoplatelets, A Model for Surface Chemistry/Optoelectronic Property Relations in Semiconductor Nanocrystals. J. Am. Chem. Soc. 2018, 140 (41), $13292-13300$.

(12) Christodoulou, S.; Climente, J. I.; Planelles, J.; Brescia, R.; Prato, M.; Martín-García, B.; Khan, A. H.; Moreels, I. Chloride-Induced Thickness Control in CdSe Nanoplatelets. Nano Lett. 2018, 18 (10), 6248-6254.

(13) Ithurria, S.; Tessier, M. D.; Mahler, B.; Lobo, R. P. S. M.; Dubertret, B.; Efros, A. L. Colloidal Nanoplatelets with Two-Dimensional Electronic Structure. Nat. Mater. 2011, 10 (12), 936-941.

(14) Shornikova, E. V.; Biadala, L.; Yakovlev, D. R.; Sapega, V. F.; Kusrayev, Y. G.; Mitioglu, A. A.; Ballottin, M. V.; Christianen, P. C. M.; Belykh, V. V.; Kochiev, M. V.; et 
al. Addressing the Exciton Fine Structure in Colloidal Nanocrystals: The Case of CdSe Nanoplatelets. Nanoscale 2018, 10 (2), 646-656.

(15) She, C.; Fedin, I.; Dolzhnikov, D. S.; Dahlberg, P. D.; Engel, G. S.; Schaller, R. D.; Talapin, D. V. Red, Yellow, Green, and Blue Amplified Spontaneous Emission and Lasing Using Colloidal CdSe Nanoplatelets. ACS Nano 2015, 9 (10), 9475-9485.

(16) Liljeroth, P.; van Emmichoven, P.; Hickey, S.; Weller, H.; Grandidier, B.; Allan, G.; Vanmaekelbergh, D. Density of States Measured by Scanning-Tunneling Spectroscopy Sheds New Light on the Optical Transitions in PbSe Nanocrystals. Phys. Rev. Lett. 2005, $95(8), 086801$.

(17) Banin, U.; Millo, O. Tunneling and Optical Spectroscopy of Semiconductor Nanocrystals. Annu. Rev. Phys. Chem. 2003, 54 (1), 465-492.

(18) Niquet, Y. M.; Delerue, C.; Allan, G.; Lannoo, M. Interpretation and Theory of Tunneling Experiments on Single Nanostructures. Phys. Rev. B - Condens. Matter Mater. Phys. 2002, $65(16), 1-14$.

(19) Hashimoto, K.; Sohrmann, C.; Wiebe, J.; Inaoka, T.; Meier, F.; Hirayama, Y.; Römer, R. A.; Wiesendanger, R.; Morgenstern, M. Quantum Hall Transition in Real Space: From Localized to Extended States. Phys. Rev. Lett. 2008, 101 (25), 256802.

(20) Ithurria, S.; Bousquet, G.; Dubertret, B. Continuous Transition from 3D to 1D Confinement Observed during the Formation of CdSe Nanoplatelets. J. Am. Chem. Soc. 2011, 133 (9), 3070-3077. 
(21) Bertrand, G. H. V.; Polovitsyn, A.; Christodoulou, S.; Khan, A. H.; Moreels, I. Shape Control of Zincblende CdSe Nanoplatelets. Chem. Commun. 2016, 52 (80), 11975-11978.

(22) Liljeroth, P.; Van Emmichoven, P. A. Z.; Hickey, S. G.; Weller, H.; Grandidier, B.; Allan, G.; Vanmaekelbergh, D. Density of States Measured by Scanning-Tunneling Spectroscopy Sheds New Light on the Optical Transitions in PbSe Nanocrystals. Phys. Rev. Lett. 2005, 95 (8).

(23) Nguyen, T. H.; Habinshuti, J.; Justo, Y.; Gomes, R.; Mahieu, G.; Godey, S.; Nys, J. P.; Carrillo, S.; Hens, Z.; Robbe, O.; et al. Charge Carrier Identification in Tunneling Spectroscopy of Core-Shell Nanocrystals. Phys. Rev. B - Condens. Matter Mater. Phys. 2011, 84 (19).

(24) Tessier, M. D.; Javaux, C.; Maksimovic, I.; Loriette, V.; Dubertret, B. Spectroscopy of Single CdSe Nanoplatelets. ACS Nano 2012, 6 (8), 6751-6758..

(25) Biadala, L.; Louyer, Y.; Tamarat, P.; Lounis, B.; Biadala, L.; Tamarat, P.; Lounis, B. Direct Observation of the Two Lowest Exciton Zero-Phonon Lines in Single CdSe/ZnS Nanocrystals. Phys. Rev. Lett. 2009, 103 (3), 037404.

(26) Ip, A. H.; Thon, S. M.; Hoogland, S.; Voznyy, O.; Zhitomirsky, D.; Debnath, R.; Levina, L.; Rollny, L. R.; Carey, G. H.; Fischer, A.; et al. Hybrid Passivated Colloidal Quantum Dot Solids. Nat. Nanotechnol. 2012, 7 (9), 577-582.

(27) Bozyigit, D.; Volk, S.; Yarema, O.; Wood, V. Quantification of Deep Traps in Nanocrystal Solids, Their Electronic Properties, and Their Influence on Device Behavior. Nano Lett. 2013, 13 (11), 5284-5288. 
(28) Pokrant, S.; Whaley, K. B. Tight-Binding Studies of Surface Effects on Electronic Structure of CdSe Nanocrystals: The Role of Organic Ligands, Surface Reconstruction, and Inorganic Capping Shells. Eur. Phys. J. D 1999, 6 (2), 255-267.

(29) Houtepen, A. J.; Hens, Z.; Owen, J. S.; Infante, I. On the Origin of Surface Traps in Colloidal II-VI Semiconductor Nanocrystals. Chem. Mater. 2017, 29 (2), 752-761.

(30) Hummon, M. R.; Stollenwerk, A. J.; Narayanamurti, V.; Anikeeva, P. O.; Panzer, M. J.; Wood, V.; Bulović, V. Measuring Charge Trap Occupation and Energy Level in CdSe/ZnS Quantum Dots Using a Scanning Tunneling Microscope. Phys. Rev. B Condens. Matter Mater. Phys. 2010, 81 (11).

(31) Biadala, L.; Peng, W.; Lambert, Y.; Kim, J. H.; Canneson, D.; Houppe, A.; Berthe, M.; Troadec, D.; Deresmes, D.; Patriarche, G.; et al. Trap-Free Heterostructure of PbS Nanoplatelets on InP(001) by Chemical Epitaxy. ACS Nano 2019, 13 (3), 1961-1967.

(32) Sun, Z.; Swart, I.; Delerue, C.; Vanmaekelbergh, D.; Liljeroth, P. Orbital and ChargeResolved Polaron States in CdSe Dots and Rods Probed by Scanning Tunneling Spectroscopy. Phys. Rev. Lett. 2009, 102 (19), 196401.

(33) Niquet, Y. M.; Delerue, C. Band Offsets, Wells, and Barriers at Nanoscale Semiconductor Heterojunctions. Phys. Rev. B - Condens. Matter Mater. Phys. 2011, 84 (7), 075478. 
(a) TEM

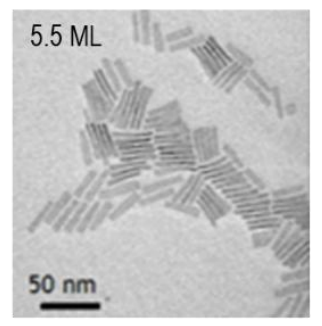

(b)
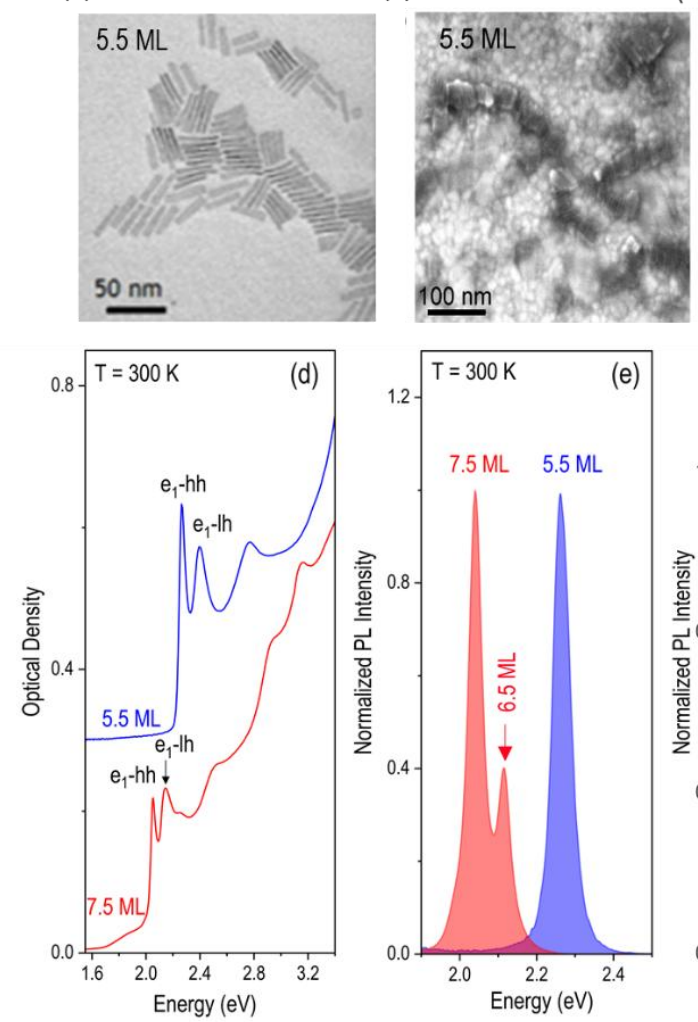

(c) AFM
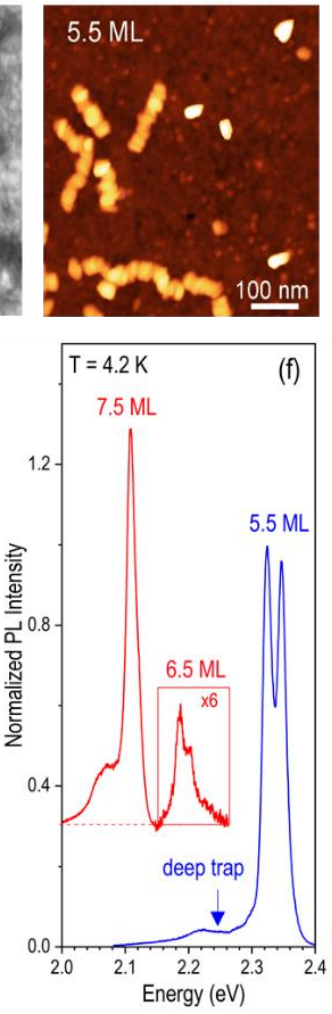

Figure 1 
(a)

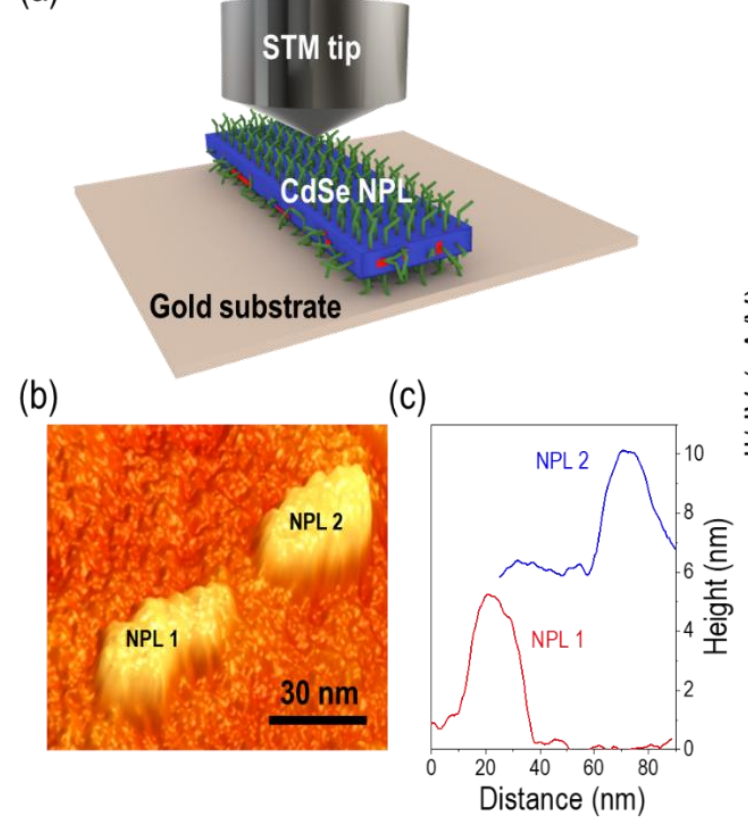

(d)

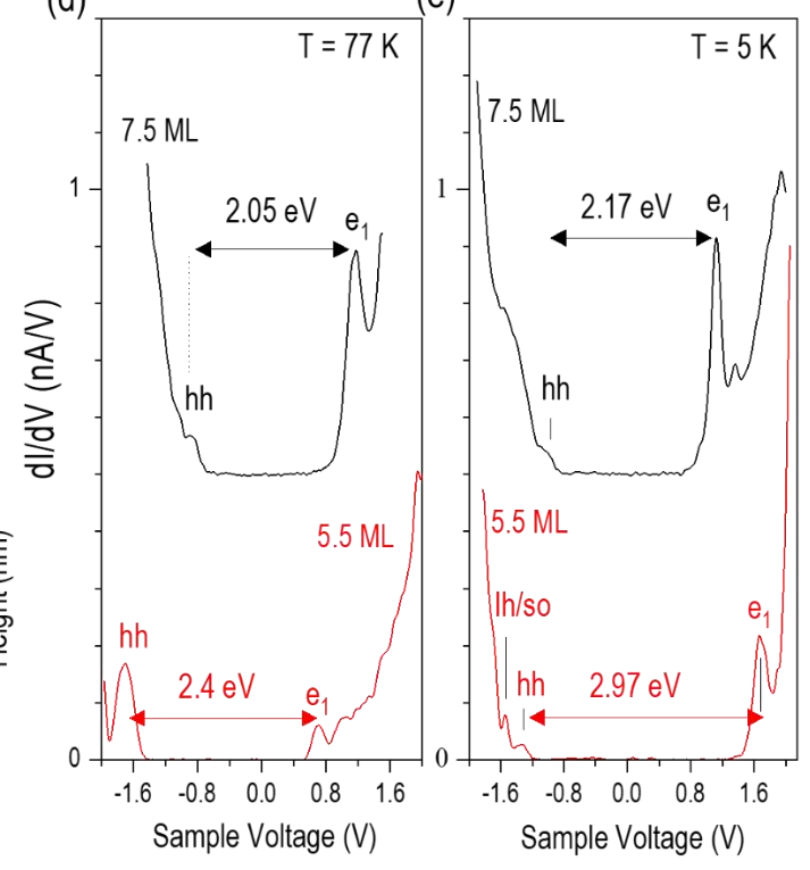

Figure 2 
(a)

(b)
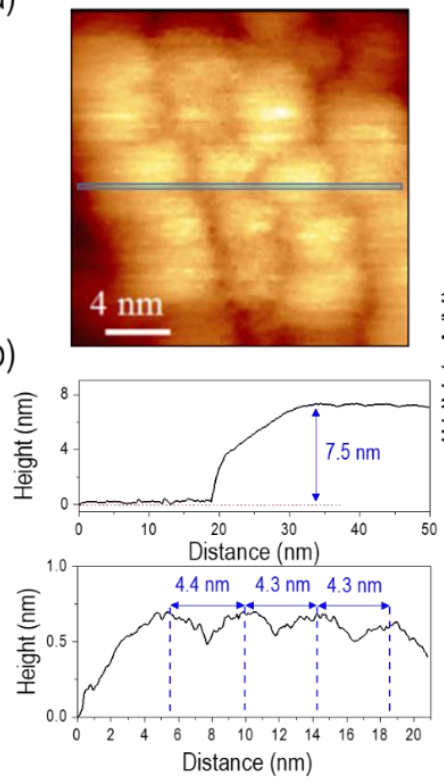

(c)

(c)

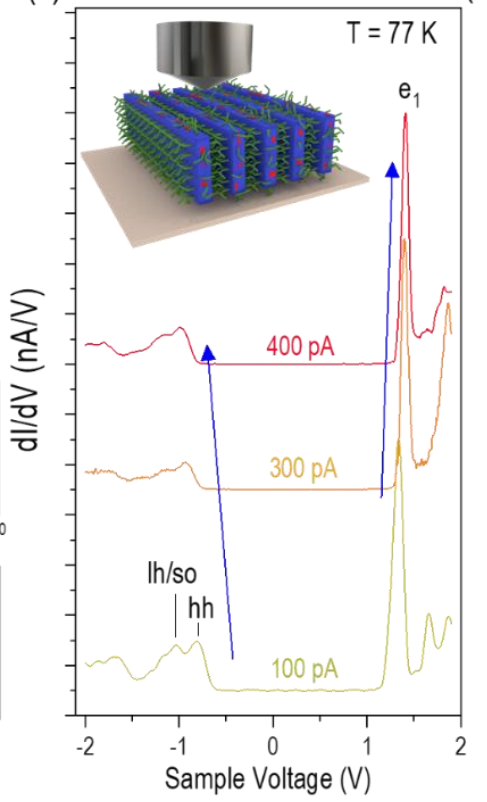

(d)

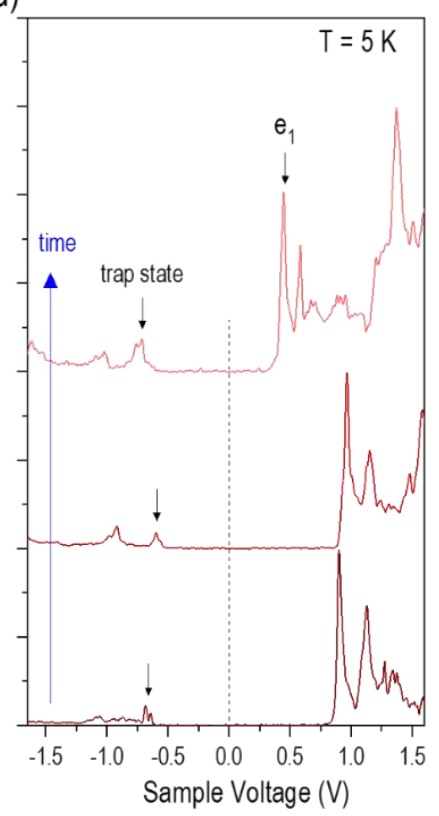

Figure 3 
(a)

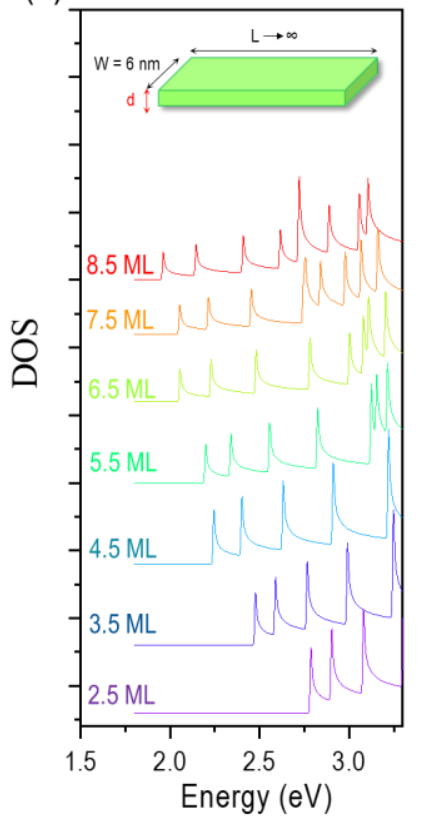

(b)

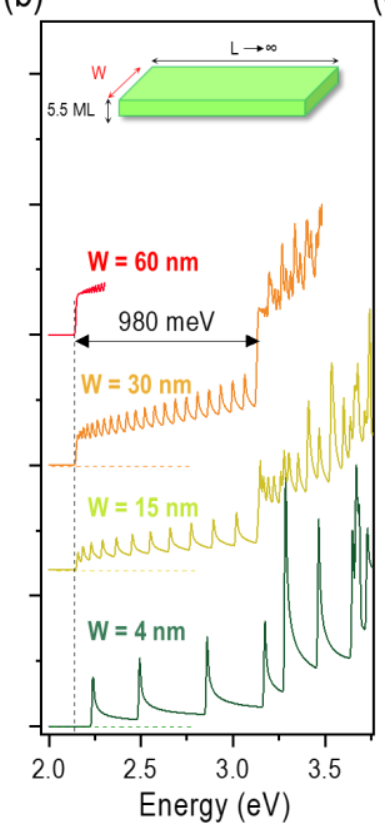

(c)

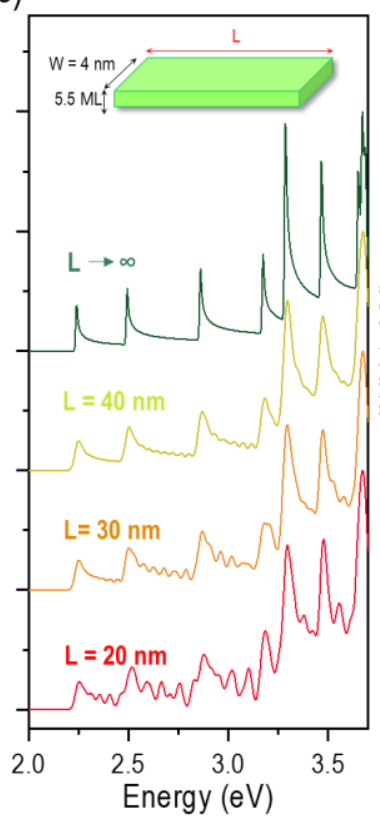

(d)

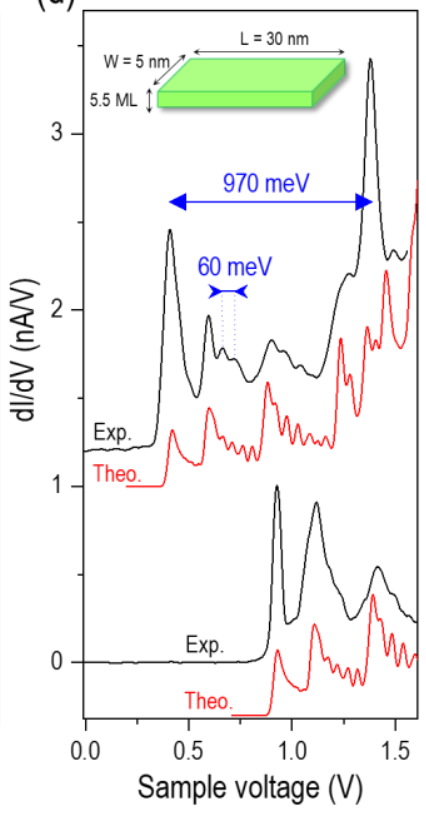

Figure 4 
TOC

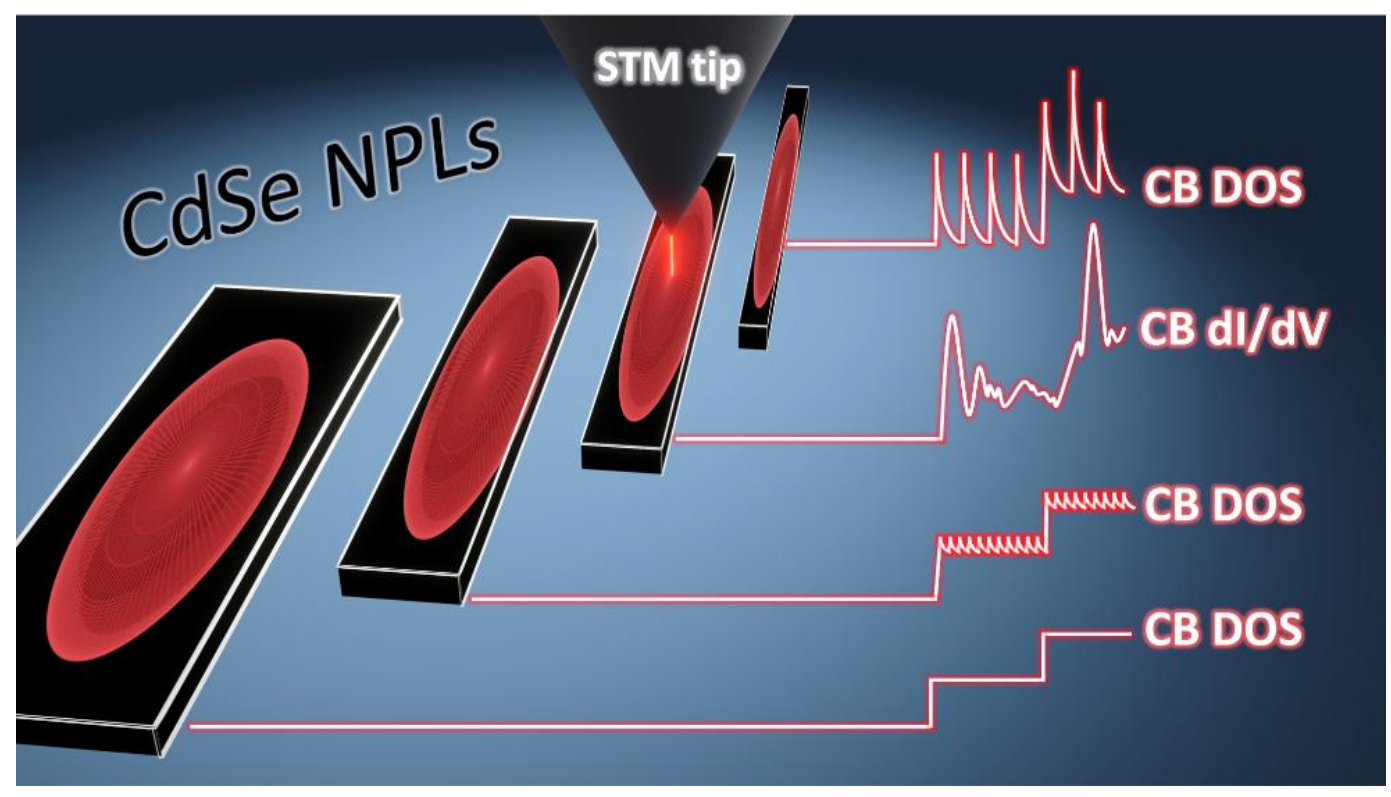

